Pacific Journal of Mathematics

DIFFERENTIABLE CURVES OF CYCLIC ORDER FOUR 


\title{
DIFFERENTIABLE CURVES OF CYCLIC ORDER FOUR
}

\section{GARY SPOAR}

\begin{abstract}
It is shown that there are only fourteen possible differentiable closed curves of cyclic order four in the real conformal plane. This classification is made with respect to numbers and types of singular points. In this regard the characteristic of a differentiable interior point of an arc and a known result of $P$. Erdös are used extensively.
\end{abstract}

In [5] N. D. Lane and P. Scherk introduced the characteristic of a differentiable interior point of an arc or curve in the real conformal plane. Using the notion of the characteristic, this paper classifies all possible simple differentiable curves of cyclic order four in the conformal plane in regard to the number and type of singular points. It is well known that such curves contain at most four singular points [9].

Moreover, differentiable curves of cyclic order four contain at most one double-point $d$ and in this case each of the loops separated by $d$ contain exactly one singular point with the characteristic $(1,1,2)$ or $(1,1,2)_{0}$; cf. $\S 5$.

In [3] C. Juel gave a similar study of such curves deriving his results using a correspondence principle dependent upon a continuous function theorem. This paper is a refinement of the Juel manuscript deriving the key global result by combining a known theorem of P. Erdös with a detailed discucssion of point osculating circles and the characteristic of such points.

In $\S 6$ a list of all possible differentiable curves of order four is given.

\section{Preliminaries.}

1.1. A point $p$ on an arc $\mathscr{C}$ is said to be (conformally) differentiable [4] if it satisfies two conditions:

I. For every point $R \neq p$ and for every sequence of points $s \rightarrow p$ on $\mathscr{A}$ there exists a circle $C_{0}$ such that $C(s, p, R) \rightarrow C_{0} . \quad C_{0}$ is called the tangent circle of $\mathscr{A}$ at $p$ through $R$ and is denoted $C\left(p^{2}, R\right)$.

II. If $s \rightarrow p$ on $\mathscr{A}$ there exists a circle $C\left(p^{3}\right)$ such that $C\left(p^{2}, s\right) \rightarrow$ $C\left(p^{3}\right) . C\left(p^{3}\right)$ is called the osculating circle of $\mathscr{A}$ at $p . C\left(p^{3}\right)$ may be the point circle $p$.

A point $p$ on $\mathscr{A}$ is said to be strongly differentiable if the following are satisfied: 
I'. Let $R \neq p, Q \rightarrow R$. If two distinct points $u, v \rightarrow p$ on . $\mathscr{\text { , }}$, then $C(u, v, Q)$ converges. on $\mathscr{2}$.

$\mathrm{II}^{\prime} . \quad C(t, u, v)$ converges if the three distinct points $t, u, v \rightarrow p$

Some results concerning differentiability are [5]:

(i) The set of all tangent circles all touch each other at $p$ (the set of all tangent circles is a pencil of the second kind with the fundamental point $p$ ).

(ii) Nontangent circles through $p$ all intersect or all support.

(iii) The nonosculating tangent circles through $p$ all intersect or all support. If $C(p) \neq p$, all of them support.

(iv) Strong differentiability implies ordinary differentiability.

(v) Strong differentiability implies that the osculating circle varies continuously with $p$.

1.2. A differentiable interior point $p$ of an arc $\mathscr{A}$ has the characteristic [5] $\left(a_{0}, a_{1}, a_{2}\right)$ if $C(p) \neq p$ or $\left(a_{0}, a_{1}, a_{2}\right)_{0}$ if $C(p)=p$ where $\left(a_{2}=\infty\right.$ will not be considered here):

(i) $a_{0}, a_{1}, a_{2}$ are equal to 1 or 2 .

(ii) $a_{0}\left[a_{0}+a_{1}\right]$ is even or odd accordingly as the nontangent circles [the nonosculating tangent circles] at $p$ support or intersect.

(iii) $a_{0}+a_{1}+a_{2}$ is even if $C\left(p^{3}\right)$ supports, odd if $C\left(p^{3}\right)$ intersects.

1.3. The cyclic order of an arc $\mathscr{A}$ is the maximum number of points in common with any circle. The order of a point $p$ is the minimum of the orders of all neighborhoods of $p$ on $\mathscr{A}$.

A point of (minimal) order three is called an ordinary point, a point of order greater than three a singular point, and a point of support of $\mathscr{A}$ with respect to $C\left(p^{3}\right)$ a vertex.

With regard to an arc or curve of cyclic order four:

(i) It contains at most four singular points [9].

(ii) No circle supports it at more than two points.

(iii) The osculating circle $C\left(p^{3}\right)$ at any singular point $p$ does not meet it again.

(iv) Points with characteristic $(1,1,1)$ are exactly the ordinary points while the singular points are vertices and have characteristic $(1,1,2),(1,1,2)_{0},(1,2,1)_{0},(2,1,1)_{0}[5]$.

(v) Points with characteristic $(1,1,1),(1,1,2)$ or $(1,1,2)_{0}$ are strongly differentiable [5].

1.4. Here a result is stated which is very useful in this discussion as well as being of some interest in its own right. An euclidean proof was originally given by P. Erdös [2], whereas a more recent conformal proof can be found in [7]. 
Let $\mathscr{R}$ be a closed simply connected region of the conformal plane bounded by a Jordan curve $\mathscr{J}$, and let $\mathscr{J}$ be divided into three arcs $\mathscr{A}_{1}, \mathscr{A}_{2}, \mathscr{A}_{3}$. Then there exists a circle contained in $\mathscr{R}$ and having points in common with all three arcs.

REMARKS. (i) $f$ divides the conformal plane into two closed simply connected regions bounded by $\mathscr{J}$. By the theorem there exist two circles, one in $\mathscr{J}_{i} \cup \mathscr{J}$ and one in $\mathscr{J}_{e} \cup \mathscr{J}\left(\mathscr{J}_{i}, \mathscr{J}_{e}\right.$ denote interior, exterior of $\mathscr{J}$, respectively) having points in common with the three arcs $\mathscr{A}_{1}, \mathscr{A}_{2}, \mathscr{A}_{3}$. We assume in our considerations that the interior of any simple curve lies at its left.

(ii) For curves of order four there are only two points of contact using (ii) of 1.3 .

1.5. A differentiable arc $\mathscr{C}$ is said to be monotone if $\mathscr{A}$ induces a unique orientation on the osculating circles at each point of $\mathscr{A}$ such that, if $p<q$ on $\mathscr{A}$,

or

$$
C\left(p^{3}\right) \subset C\left(q^{3}\right)_{i} \text { and } C\left(q^{3}\right) \subset C\left(p^{3}\right)_{e}
$$

$$
C\left(p^{3}\right) \subset C\left(q^{3}\right)_{e} \text { and } C\left(q^{3}\right) \subset C\left(p^{3}\right)_{i} .
$$

(i) Arcs of order three are monotone [6].

(ii) Let each interior point of an arc $\%_{4}$ of order four be ordinary. Then the closed are $\overline{\mathscr{A}}_{4}$ is monotone.

Proof. Each interior point of $\mathscr{A}_{4}$ is ordinary. Also end points of $\mathscr{A}_{4}$ are ordinary [8]. Hence, each [interior] point of the arc posesses a [two-sided] neighborhood of order three. By (i) each of these neighborhoods is monotone. By taking the union of these neighborhoods the monotony of $\overline{\mathscr{A}}_{4}$ is obtained.

2. Induced orientations on the osculating circle.

2.1. A fixed direction on an arc $\mathscr{A}$ of order three defines the same orientation at each arc point. A point $p$ which is the end

point of two order three arcs may have the configulation

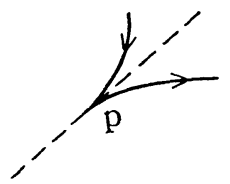

in which case the orientation is the same as the point moves from

one arc to the other. If the arcs meet in the form

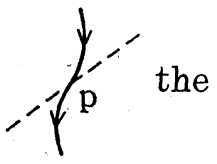


orientation changes (cf. Figures 1-4 where $p$ is not chosen as the point at infinity and the point of infinity is removed to give the open plane; the separating tangent line to the arc $p$, considered as a circle, is a member of the pencil of all tangent circles of the arc at $p$ ).

2.2. Let $p$ be a point of a differentiable curve $\mathscr{C}_{4}$ of order four with the characteristic $(2,1,1)_{0}$ (cf. Figures 1 and 2). Since $p$ is a cusp point, we may assume that each circle that supports $\mathscr{C}_{4}$ at $p$ lies locally on the same side of $\mathscr{C}_{4}$ outside $p$. Let

$$
N=N^{\prime} \cup\{p\} \cup N^{\prime \prime}
$$

be a small two-sided neighborhood of $p$ on $\mathscr{C}_{4}$ where $N^{\prime}\left[N^{\prime \prime}\right]$ is a preceding [proceeding] neighborhood of $p$.

We know that the osculating circles $C^{\prime}\left(p^{3}\right), C^{\prime \prime}\left(p^{3}\right)$ of $N^{\prime} \cup\{p\}$, $N^{\prime \prime} \cup\{p\}$, respectively, are each the point circle $p$ since $N$ is differentiable at $p$ and $p$ has the characteristic $(2,1,1)_{0}$.

We would like to know in what manner $N^{\prime} \cup\{p\}$ and $N^{\prime \prime} \cup\{p\}$ induce orientations on their common osculating circle

$$
C^{\prime}\left(p^{3}\right)=C^{\prime \prime}\left(p^{3}\right)=p \text {. }
$$

At a point with the characteristic $(2,1,1)_{0}$ the orientation remains the same as we move from one arc to the other with the result that

$$
\begin{aligned}
& C^{\prime}\left(p^{3}\right)_{i}=C^{\prime \prime}\left(p^{3}\right)_{i}=\varnothing \quad \text { or } \\
& C^{\prime}\left(p^{3}\right)_{e}=C^{\prime \prime}\left(p^{3}\right)_{e}=\varnothing
\end{aligned}
$$

depending only on the way $\mathscr{C}_{4}$ was originally oriented (recalling that the interior lies at the left of a curve or circle).
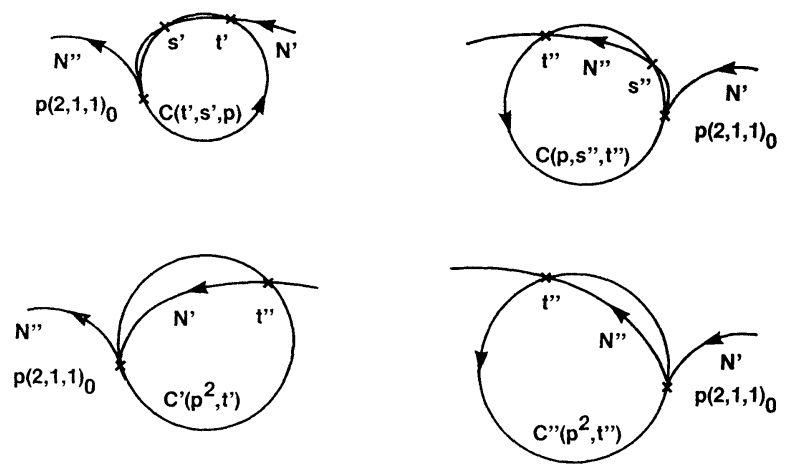

FiguRe 1

FIGURe 2

2.3. Now let $p$ be a point of a differentiable curve $\mathscr{C}_{4}$ of order four with the characteristic $(1,2,1)_{0}$ (cf. Figures 3 and 4). Again let $N$ be a two-sided neighborhood of $p$ on $\mathscr{C}_{4}$ as in 2.2 . 


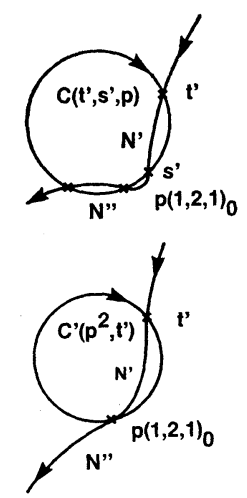

Figure 3

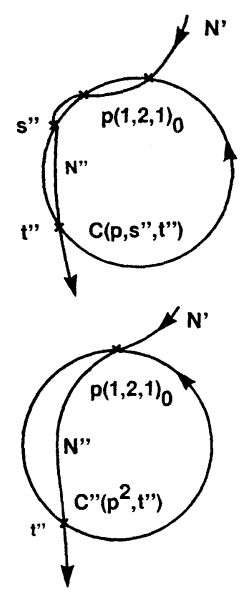

FIGURE 4

At a point with the characteristic $(1,2,1)_{0}$ the orientation changes as we move from one arc to the other with the result that either

$$
\begin{array}{lll}
C^{\prime}(p)_{i}=\varnothing, & C^{\prime \prime}(p)_{e}=\varnothing & \text { or } \\
C^{\prime}(p)_{e}=\varnothing, & C^{\prime \prime}(p)_{i}=\varnothing &
\end{array}
$$

again depending only on the given orientation of $\mathscr{C}_{4}$.

2.4. We note that from the above, if $p$ is of the type $(2,1,1)_{0}$, then the natural orientation of $C^{\prime}\left(p^{3}\right)=p$ is the same as the induced orientation of $C^{\prime \prime}\left(p^{3}\right)=p$. However, if $p$ has the characteristic $(1,2,1)_{0}$, we see that the induced orientation of $C^{\prime}\left(p^{3}\right)=p$ is opposite to the natural one induced on $C^{\prime \prime}\left(p^{3}\right)=p$.

Thus the natural orientation induced by $\mathscr{C}_{4}$ on the osculating circles at the points of $N$ is discontinuous at $p$ in the case where the characteristic of $p$ is $(1,2,1)_{0}$.

2.5. Let $p$ be a point of $\mathscr{C}_{4}$ with the characteristic one of $(1,1,1)$, $(1,1,2)$ or $(1,1,2)_{0}$. Now $p$ is a strongly differentiable point and the natural orientations induced on the two one-sided osculating circles of $\mathscr{C}_{4}$ at $p$ are identical. Moreover, this orientation varies continuously as one passes through $p$ on $\mathscr{C}_{4}$.

2.6. Analogous considerations to 2.2 and 2.3 of points with the characteristic $(2,1,1)_{0}$ or $(1,2,1)_{0}$ in analytic euclidean geometry give:

(i) in the case of a point with the characteristic $(2,1,1)_{0}$ the two one-sided curvatures are both $+\infty$ or are both $-\infty$.

(ii) for a point with the characteristic $(1,2,1)_{0}$ the two one-sided curvatures are $+\infty,-\infty$ or are $-\infty,+\infty$. 
3. Number and type of singular points on simple $\mathscr{C}_{4}$ 's.

LEMMA 3.1. $\mathscr{C}_{4}$ contains at most one point with the characteristic $(2,1,1)_{0}$.

Proof. Suppose that $\mathscr{C}_{4}$ contains at least two points $q_{1}, q_{2}$ with characteristic $(2,1,1)_{0}$. Let $r \in \mathscr{C}_{4}, r \neq q_{1}, q_{2}$ and $K=K\left(q_{1}, q_{2}, r\right)$ the circle determined by $q_{1}, q_{2}$ and $r$. Counting multiplicities, at most one of $q_{1}, q_{2}$ and $r$ is a point of support of $K$ with $\mathscr{C}_{4}$. Otherwise, a circle close to $K$ would meet $\mathscr{C}_{4}$ at least five times.

If $K$ supports $\mathscr{C}_{4}$ at $r$, then $K$ intersects $\mathscr{C}_{4}$ at $q_{1}, q_{2}$. Since $q_{1}$ has characteristic $(2,1,1)_{0}$, then $K$ is a tangent circle of $\mathscr{C}_{4}$ at $q_{1}$ which intersects $\mathscr{C}_{4}$ there. Thus a circle close to $K$ would meet $\mathscr{C}_{4}$ three times close to $q_{1}$ and at points close to $q_{2}$ and $r$, contradiction.

If $K$ supports $\mathscr{C}_{4}$ at $q_{1}$, then similarly $K$ is a tangent circle of $\mathscr{C}_{4}$ at $q_{2}$ intersecting $\mathscr{C}_{4}$ there and a contradiction is obtained as above.

Thus $q_{1}, q_{2}$ and $r$ are all points of intersection of $K$ with $\mathscr{C}_{4}$ and hence $K$ is a tangent circle of $\mathscr{C}_{4}$ at $q_{1}$ intersecting $\mathscr{C}_{4}$ there giving rise to a contradiction of the order of $\mathscr{C}_{4}$ again as above.

LEMMA 3.2. $\mathscr{C}_{4}$ contains at most two points with the characteristic $(1,2,1)_{0}$.

Proof. Suppose $\mathscr{C}_{4}$ contains three points $p_{1}, p_{2}, p_{3}$ with the characteristic $(1,2,1)_{0}$. Then these points divide $\mathscr{C}_{4}$ into three closed arcs. Hence by 1.4 there exists a circle $K$ lying in $\mathscr{C}_{4} \cup \mathscr{C}_{4 i}$, having points in common with all three arcs. By (ii ) of 1.4, one of the $p_{i}$, say $p$ is a point of contract of $K$ with $\mathscr{C}_{4}$. Hence $K$ supports $\mathscr{C}_{4}$ at $p$. But this possibility is excluded by the characteristic of $p$, since both the nonosculating tangent circles and the nontangent circles of $\mathscr{C}_{4}$ at $p$ intersect $\mathscr{C}_{4}$ at $p$.

LEMMA 3.3. If $\mathscr{C}_{4}$ contains a point with the characteristic $(2,1,1)_{0}$, then at most one point of $\mathscr{C}_{4}$ has the characteristic $(1,2,1)_{0}$.

Proof. Let $p$ be a point of $\mathscr{C}_{4}$ with characteristic $(2,1,1)_{0}$ and $q_{1}, q_{2}$ points of $\mathscr{C}_{4}$ with characteristic $(1,2,1)_{0}$. Then $p, q_{1}, q_{2}$ divide $\mathscr{C}_{4}$ into three closed arcs. By 1.4 there exists a circle $K$ lying in $\mathscr{C}_{4} \cup \mathscr{C}_{4}$, having points in common with all three arcs. Again, one of the points $p, q_{1}, q_{2}$ is a point of contact of $K$ with $\mathscr{C}_{4}$. This point of $K$ cannot be either $q_{1}$ or $q_{2}$. Otherwise, $K$ would support $\mathscr{C}_{4}$ at this point. But this situation is excluded by the characteristic, since both the nonosculating tangent circles and the nontangent 
circles of $\mathscr{C}_{4}$ intersect $\mathscr{C}_{4}$ at a point of type $(1,2,1)_{0}$. Hence $p$ must be the point of contact of $K$ with $\mathscr{C}_{4}$.

However, by the Remark (i) following 1.4, there exists a circle $K^{*}$ lying in $\mathscr{C}_{4} \cup \mathscr{C}_{4_{e}}$, having points in common with all three arcs. As before, neither $q_{1}$ or $q_{2}$ is a point of contact of $K^{*}$ with $\mathscr{C}_{4}$. Hence $p$ is again the point of contact of $K^{*}$ with $\mathscr{C}_{4}$. Now we have two circles $K, K^{*}$ which support $\mathscr{C}_{4}$ at $p$ and lie on opposite sides of $\mathscr{C}_{4}$, outside $p$. But this is impossible, since $p$ is a cusp point. Thus we have the desired result.

Lemma 3.4. A differentiable $\mathscr{C}_{4}$ contains at least two singular points.

Proof. If $\mathscr{C}_{4}$ contains no singular points, then $\mathscr{C}_{4}$ is monotone by (ii) of 1.5 , contradiction.

Let $\mathscr{A}_{4}=\mathscr{C}_{4} \mid\{p\}$ where $p$ is a singular point. If each point of $\mathscr{A}_{4}$ is ordinary then $\overline{\mathscr{A}}_{4}=\mathscr{C}_{4}$ is monotone, contradiction.

Thus $\mathscr{C}_{4}$ contains at least two singular points.

LeMMA 3.5. A differentiable $\mathscr{C}_{4}$ with one point with the characteristic $(1,2,1)_{0}$ contains exactly three singular points.

Proof. Let $p$ be the point with characteristic $(1,2,1)_{0}$. By (i) of 1.3 and $3.4, \mathscr{C}_{4}$ contains two, three or four singular points.

Assume that $\mathscr{C}_{4}$ contains one other singular point $q$. Then $q$ has the characteristic one of $(1,1,2),(1,1,2)_{0}$ or $(2,1,1)_{0}$. Also the ares $\overline{p q}$ and $\overline{q p}$ are monotone, by (ii) of 1.6. Note that the orientation of the osculating circle is continuous at $q$, by 2.2 and 2.5 but discontinuous at $p$ by 2.3 .

Call $C^{\prime}\left(p^{3}\right), C^{\prime \prime}\left(p^{3}\right),\left[C^{\prime}\left(q^{3}\right), C^{\prime \prime}\left(q^{3}\right)\right]$ the two one-sided osculating circles at $p$ [at $q$ ] as in $\S 2$ and assume that $C^{\prime \prime}\left(p^{3}\right)_{i}=\varnothing$ (the case $C^{\prime \prime}\left(p^{3}\right)_{e}=\varnothing$ follows similarly).

Now $\overline{p q}$ is monotone. Hence,

and

$$
C^{\prime}\left(q^{3}\right) \subset C^{\prime \prime}\left(p^{3}\right)_{e}
$$

$$
C^{\prime \prime}\left(p^{3}\right) \subset C^{\prime}\left(q^{3}\right)_{i}=C^{\prime \prime}\left(q^{3}\right)_{i} .
$$

Thus $p \in C^{\prime \prime}\left(q^{3}\right)_{i}$ and $\mathscr{C}_{4} \mid\{q\} \subset C^{\prime \prime}\left(q^{3}\right)_{i}$ by (iii) of 1.3 . Next $\overline{q p}$ is monotone. Hence

and

$$
C^{\prime}\left(p^{3}\right) \subset C^{\prime \prime}\left(q^{3}\right)_{i}
$$

$$
C^{\prime \prime}\left(q^{3}\right) \subset C^{\prime}\left(p^{3}\right)_{e}=C^{\prime \prime}\left(p^{3}\right)_{i}=\varnothing ;
$$

contradiction. 
The case where $\mathscr{C}_{4}$ contains three other singular points $q$ with the characteristic $(1,1,2)(1,1,2)_{0}$ or $(2,1,1)_{0}$ follows similarly; the important point is that there are an odd number of $q$ 's and the orientation is continuous at these points.

Corollary. A differentiable $\mathscr{C}_{4}$ with two points with the characteristic $(1,2,1)_{0}$ contains either two or four singular points.

Proof. The proof follows in an analogous manner to the theorem using the discontinuity of the orientation of the osculating circle at the $(1,2,1)_{0}$ points.

LeMma 3.6. A differentiable $\mathscr{C}_{4}$ with no $(1,2,1)_{0}$ points contains either two or four singular points.

Proof. Since there are only singular points with the characteristic $(1,1,2),(1,1,2)_{0}$ or $(2,1,1)_{0}$, the orientation of the osculating circle at these points is continuous. Hence $\mathscr{C}_{4}$ must contain an even number of singular points.

4. Impossible $\mathscr{C}_{4}^{\prime}$ 's.

LEMMA 4.1. There is no differentiable $\mathscr{C}_{4}$ with four singular points including one with characteristic $(2,1,1)_{0}$.

Proof. Firstly $\mathscr{C}_{4}$ contains only one point $p$ with characteristic $(2,1,1)_{0}$, by 3.1 and none with characteristic $(1,2,1)_{0}$, by 3.3 and 3.5. Thus the three other singular points $p<q_{1}<q_{2}<q_{3}$ have characteristic $(1,1,2)$ or $(1,1,2)_{0}$. Assume that each of the $q_{i}$ are $(1,1,2)$ points. The other cases similarly lead to a contradiction.

Either $C\left(p^{3}\right)_{e}=\varnothing$ or $C\left(p^{3}\right)_{i}=\varnothing$, say the former. Using (iii) of 1.3, the continuity of the orientation of the osculating circle at each point and the monotony of the arcs $\overline{p q_{1}}$ and $\overline{q_{3} p}$, one obtains

and

$$
\begin{aligned}
& C\left(q_{1}^{3}\right) \backslash\left\{q_{1}\right\} \subset \mathscr{C}_{{ }_{4 i}} \\
& \mathscr{C}_{4} \subset C\left(q_{1}^{3}\right)_{e}
\end{aligned}
$$

$$
\begin{aligned}
& C\left(q_{3}^{3}\right) \backslash\left\{q_{3}\right\} \subset \mathscr{C}_{4 i} \\
& \mathscr{C}_{4} \subset C\left(q_{3}^{3}\right)_{e} .
\end{aligned}
$$

Now $\mathscr{C}_{4}$ is separated into three closed arcs by the points $p, q_{1}, q_{3}$. By 1.4 , there is a circle $K$ lying in $\mathscr{C}_{4} \cup \mathscr{C}_{4 i}$ having points in common with all three ares. By (ii) of 1.4 one of $p, q_{1}, q_{3}$ is a point of contact. The point $p$ has already been excluded since $C\left(p^{3}\right)_{e}=\varnothing$ (see first paragraph of 2.2). Hence one of $q_{1}, q_{3}$, say $q_{1}$ is a point 


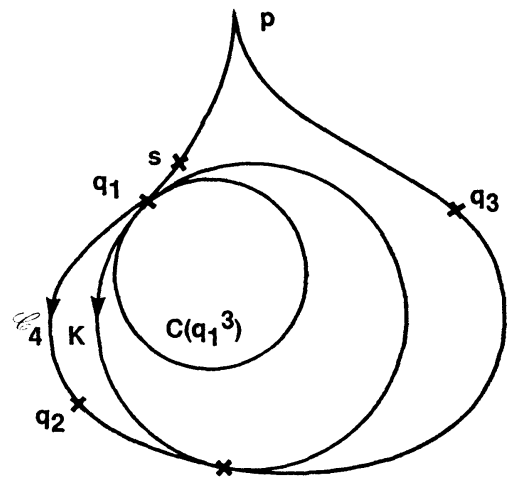

FIGURE 5

of contact of $K$ with $\mathscr{C}_{4}$ and $K$ is a tangent circle of $\mathscr{C}_{4}$ at $q_{1}$. But then (see Figure 5) if $s$ is close to $q_{1}$ on $\mathscr{C}_{4} C\left(q_{1}^{2}, s\right)$ and $C\left(q_{1}^{3}\right)$ are separated by $K$ and $C\left(q_{1}^{2}, s\right)$ cannot converge to $C\left(q_{1}^{3}\right)$ as $s \rightarrow q_{1}$ on $\mathscr{C}_{4}$. This is impossible since $q_{1}$ is a differentiable point.

The case where $C\left(p^{3}\right)_{i}=\varnothing$ gives rise to a similar contradiction where a circle $K^{*}$ lying in $\mathscr{C}_{4} \cup \mathscr{C}_{4_{e}}$ (see Remark (i) to 1.4) having $q_{1}$ or $q_{3}$ as a point of contact is used instead of $K$ as above.

LEMma 4.2. No differentiable $\mathscr{C}_{4}$ exists with more than two singular points including two with characteristic $(1,2,1)_{0}$.

Proof. By 3.2, 3.3 and the corollary to $3.5, \mathscr{C}_{4}$ contains exactly two points $p_{1}, p_{2}$ with characteristic $(1,2,1)_{0}$ and two other points $q_{1}, q_{2}$ with characteristic $(1,1,2)$ or $(1,1,2)_{0}$. Assume that $q_{1}, q_{2}$ are $(1,1,2)$ points and $C\left(q_{1}^{3}\right) \subset \mathscr{C}_{4 i}$. The other cases can be handled in a similar manner by appropriately picking $K$ or $K^{*}$ as in 4.1.

The points $p_{1}, p_{2}, q_{1}$ separate $\mathscr{C}_{4}$ into three closed arcs. Hence there is a circle $K$ lying in $\mathscr{C}_{4} \cup \mathscr{C}_{4 i}$ having $q_{1}$ as a point of contact (the points $p_{1}, p_{2}$ cannot be contact points as in 3.2). Again as in 4.1, for $s$ close to $q_{1}$ on $\mathscr{C}_{4}, C\left(q_{1}^{2}, s\right)$ and $C\left(q_{1}^{3}\right)$ are separated by $K$ and hence $C\left(q_{1}^{2}, s\right)$ cannot converge to $C\left(q_{1}^{3}\right)$ as $s \rightarrow q_{1}$ on $\mathscr{C}_{4}$.

Similarly as in 4.1 and 4.2 one obtains:

LEMMA 4.3. No differentiable $\mathscr{C}_{4}$ exists with exactly three singular points including one point with characteristic $(1,2,1)_{0}$ and one with characteristic $(2,1,1)_{0}$.

\section{Double-points.}

LeMma 5.1. $\mathscr{C}_{4}$ contains at most one double-point. (A point where $\mathscr{C}_{4}$ meets itself.) 
Proof. Suppose $\mathscr{C}_{4}$ contains at least two double-points $d_{1}, d_{2}$. Take any other point $s$ on $\mathscr{C}_{4}$ and consider the circle $K=K\left(d_{1}, d_{2}, s\right)$. A circle close to $K$ will meet $\mathscr{C}_{4}$ at two points close to $d_{1}$, at two points close to $d_{2}$ and at one point close to $s$, contradiction.

In the sequel let $\mathscr{C}_{4}$ be a differentiable curve of order four with one double-point $d$ separating $\mathscr{C}_{4}$ into two simple arcs $\mathscr{L}_{1}, \mathscr{L}_{2}$ called loops. Certainly $d$ is simple in the sense that $\mathscr{C}_{4}$ passes through it exactly twice.

Lemma 5.2. The point $d$ is not singular.

Proof. In all cases whether $d$ has the characteristic $(1,1,2)$, $(1,1,2)_{0},(1,2,1)_{0}$ or $(2,1,1)_{0}$ on one of the arcs $\mathscr{A}$ of $\mathscr{C}_{4}$ passing through $d$, a circle close to the osculating circle of $\mathscr{C}$ at $d$ will intersect $\mathscr{A}$ at four distinct points as well as at another point on the other arc of $\mathscr{C}_{4}$ passing through $d$. This contradicts the order of $\mathscr{C}_{4}$.

Lemma 5.3. $\mathscr{C}_{4}$ contains at least two singular points, one in each of the loops determined by $d$.

Proof. Suppose $\mathscr{L}_{1}$ contains no singular points. Then $\mathscr{L}_{1} \cup\{d\}$ is monotone by (ii) of 1.5 , contradiction.

Lemma 5.4. $\mathscr{C}_{4}$ contains at most two singular points (hence exactly one in each loop).

Proof. Suppose there are at least three singular points. Then there are at least two $p_{1}, p_{2}$ in one of the loops, $\mathscr{L}_{1}$ say, with the subarc $\bar{p}_{1} p_{2}$ of $\mathscr{L}_{1}$ monotone. But then as $s$ moves continuously and monotonically on $\overline{p_{1} p_{2}}$ from $p_{1}$ to $p_{2}$ the osculating circle $C\left(s^{3}\right)$ of $\mathscr{L}_{1}$ at $s$ intersects $\mathscr{C}_{4}$ at a point $u$ which moves monotonically and continuously on $\mathscr{C}_{4}$ in the opposite direction from $p_{1}$ back to $p_{2}$. Hence there is a point $s^{*}$ between $p_{1}$ and $p_{2}$ where $C\left(s^{* 3}\right)$ passes through $d$. But then a circle close to $C\left(s^{* 3}\right)$ will intersect $\mathscr{L}_{1}$ at three points close to $s^{*}$ and at two points close to $d$, contradiction.

LEMMA 5.5. $\mathscr{C}_{4}$ contains no points with the characteristic $(2,1,1)_{0}$ or $(1,2,1)_{0}$.

Proof. Let $p$ be a singular point with the characteristic $(2,1,1)_{0}$ or $(1,2,1)_{0}$. Then the tangent circle $C\left(p^{2}, d\right)$ of $\mathscr{C}_{4}$ at $p$ passing through $d$ intersects $\mathscr{C}_{4}$ at $p$ [3]. But then a circle close to $C\left(p^{2}, d\right)$ will intersect $\mathscr{C}_{4}$ at three points close to $p$ and at two points close 
to $d$, contradiction.

Lemmas 5.1-5.5 give the following.

THEOREM. Let $\mathscr{C}_{4}$ be a differentiable curve of cyclic order four with double-points. Then $\mathscr{C}_{4}$ contains exactly one double-point $d$ which is simple and not singular. Moreover, $\mathscr{C}_{4}$ is a strongly differentiable curve composed of exactly two loops each containing exactly one singular point with the characteristic $(1,1,2)$ or $(1,1,2)_{0}$.

6. Possible differentiable $\mathscr{C}_{4}$ 's. The results of $\S \S 3,4$ and 5 give exactly fourteen possible differentiable curves of cyclic order four. These are listed in the chart (here we have taken the point at infinity not on $\mathscr{C}_{4}$ and removed this point to get the open plane).

SIMPLE $\mathscr{C}_{4}{ }^{\prime}$ 's

\begin{tabular}{|c|c|c|}
\hline Number & Distribution & General Type \\
\hline 4 & $\begin{array}{l}4(1,1,2) \\
3(1,1,2), 1(1,1,2)_{0} \\
2(1,1,2), 2(1,1,2)_{0} \\
1(1,1,2), 3(1,1,2)_{0} \\
4(1,1,2)_{0}\end{array}$ & 3 or 0 \\
\hline 3 & $\begin{array}{l}1(1,2,1)_{0}, 2(1,1,2) \\
1(1,2,1)_{0}, 1(1,1,2), 1(1,1,2)_{0} \\
1(1,2,1)_{0}, 2(1,1,2)_{0}\end{array}$ & $\begin{array}{l}\text { or } \\
\text { or }\end{array}$ \\
\hline 2 & $\begin{array}{l}1(2,1,1)_{0}, 1(1,1,2) \\
1(2,1,1)_{0}, 1(1,1,2)_{0} \\
2(1,2,1)_{0}\end{array}$ & or \\
\hline \multicolumn{3}{|c|}{$\mathscr{C}_{4}^{\prime}$ 's WITH ONE DOUBLE-POINT } \\
\hline 2 & $\begin{array}{l}2(1,1,2) \\
1(1,1,2),(1,1,2)_{0} \\
2(1,1,2)_{0}\end{array}$ & $\begin{array}{lll}\ominus & \text { or } & \varrho \\
0 & \text { or } & \ddots \\
\text { or } & & \theta\end{array}$ \\
\hline
\end{tabular}

\section{REFERENCES}

1. O. Haupt and H. Künneth, Geometrische Ordnungen, Springer-Verlag, Berlin, 1967.

2. S. B. Jackson, Vertices for plane curves, Bull. Amer. Math. Soc., 50 (1944), 564-578. 
3. C. Juel, On Simple Cykliske Kurver, D. Kgl. Danske Vidensk. Skrifter, 7-Raekke, Naturvidensk. og Matem. Afd. VIII 6, Copenhagen, 1911.

4. N.D. Lane and P. Scherk, Differentiable points in the conformal plane, Canad. J. Math., 5 (1952), 512-518.

5. - Characteristic and order of differentiable points in the conformal plane, Trans. Amer. Math. Soc., 81 (1956), 358-378.

6. N. D. Lane, K.D. Singh and P. Scherk, Monotony of the osculating circles of arcs of cyclic order three, Canad. Math. Bull., 7 No. 2 (1964).

7. N. D. Lane and G. Spoar, A conformal proof of a Jordan curve problem, Canad. Math. Bull., 12 No. 5 (1969).

8. - On singular points of normal arcs of cyclic order four, Canad. Math. Bull., 17 (3) (1974), 391-396.

9. G. Spoar, $A$ least upper bound for the number of singular points on normal arcs and curves of cyclic order four, Geometriae Dedicata, 7 (1978), 37-43.

Received December 2, 1977 and in revised form March 9, 1981,

UNIVERSITY OF GUELPH

Guelph, Ontario, Canada 


\section{PACIFIC JOURNAL OF MATHEMATICS}

\section{EDITORS}

DONALD BABBITT (Managing Editor)

University of California

Los Angeles, California 90024

HUGO ROSSI

University of Utah

Salt Lake City, UT 84112

C. C. MOORE and ARTHur AGUS

University of California

Berkeley, CA 94720
J. DUGUNDJI

Department of Mathematics

University of Southern California

Los Angeles, California 90007

R. FINN and J. MILGRAM

Stanford University

Stanford, California 94305

\section{ASSOCIATE EDITORS}
R. Arnes
E. F. BECKENBACH
B. H. NeumanN
F. WOLF
K. YösHIDA

\section{SUPPORTING INSTITUTIONS}

UNIVERSITY OF ARIZONA

UNIVERSITY OF BRITISH COLUMBIA

CALIFORNIA INSTITUTE OF TECHNOLOGY

UNIVERSITY OF CALIFORNIA

MONTANA STATE UNIVERSITY

UNIVERSITY OF NEVADA, RENO

NEW MEXICO STATE UNIVERSITY

OREGON STATE UNIVERSITY
UNIVERSITY OF OREGON

UNIVERSITY OF SOUTHERN CALIFORNIA

STANFORD UNIVERSITY

UNIVERSITY OF HAWAII

UNIVERSITY OF TOKYO

UNIVERSITY OF UTAH

WASHINGTON STATE UNIVERSITY

UNIVERSITY OF WASHINGTON 


\section{Pacific Journal of Mathematics}

Vol. 102, No. $1 \quad$ January, 1982

S. Agou, Degré minimum des polynômes $f\left(\sum_{i=0}^{m} a_{i} X^{p^{r i}}\right)$ sur les corps finis

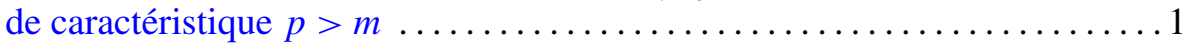

Chi Cheng Chen, On the image of the generalized Gauss map of a complete

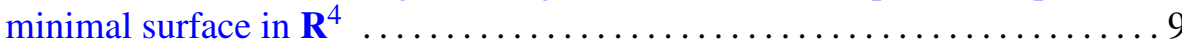

Thomas Curtis Craven and George Leslie Csordas, On the number of real

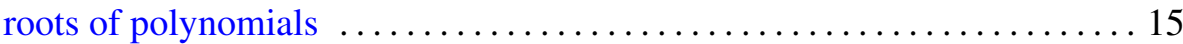

Allan L. Edelson and Kurt Kreith, Nonlinear relationships between oscillation and asymptotic behavior ....................... 29

B. Felzenszwalb and Antonio Giambruno, A commutativity theorem for

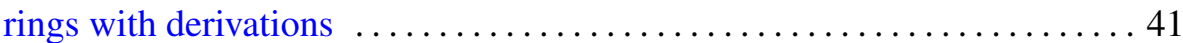

Richard Elam Heisey, Manifolds modelled on the direct limit of lines . . . . . 47

Steve J. Kaplan, Twisting to algebraically slice knots $\ldots \ldots \ldots \ldots \ldots \ldots 5$

Jeffrey C. Lagarias, Best simultaneous Diophantine approximations. II.

Behavior of consecutive best approximations $\ldots \ldots \ldots \ldots \ldots \ldots \ldots 61$

Masahiko Miyamoto, An affirmative answer to Glauberman's conjecture . . . 889

Thomas Bourque Muenzenberger, Raymond Earl Smithson and L. E.

Ward, Characterizations of arboroids and dendritic spaces ........... 107

William Leslie Pardon, The exact sequence of a localization for Witt

groups. II. Numerical invariants of odd-dimensional surgery

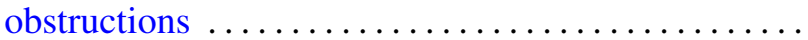

Bruce Eli Sagan, Bijective proofs of certain vector partition identities

Kichi-Suke Saito, Automorphisms and nonselfadjoint crossed products ...

John Joseph Sarraille, Module finiteness of low-dimensional PI rings ...

Gary Roy Spoar, Differentiable curves of cyclic order four . .

William Charles Waterhouse, Automorphisms of quotients of $\Pi \mathrm{GL}\left(n_{i}\right)$

Leslie Wilson, Mapgerms infinitely determined with respect to right-left equivalence

Rahman Mahmoud Younis, Interpolation in strongly logmodular

algebras 